\title{
Análise dos fatores associados a mortalidade da COVID-19 em uma cidade de tríplice fronteira
}

\author{
Analysis of factors associated with COVID-19 mortality in a triple border city \\ Análisis de factores asociados a la mortalidad por COVID-19 en una ciudad de triple frontera
}

Recebido: 30/11/2021 | Revisado: 08/12/2021 | Aceito: 17/12/2021 | Publicado: 01/01/2022

\author{
William da Costa Moreira \\ ORCID: https://orcid.org/0000-0001-6685-3549 \\ Centro Universitário Dinâmica das Cataratas, Brasil \\ E-mail: williamscorpion10@gmail.com \\ Wesley Martins \\ ORCID: https://orcid.org/0000-0003-1083-9515 \\ Centro Universitário Dinâmica das Cataratas, Brasil \\ E-mail: wesley.martins@udc.edu.br
}

\begin{abstract}
Resumo
Este estudo tem como objetivo analisar os fatores associados a mortalidade pela COVID-19 em uma cidade da tríplice fronteira. Trata-se de uma pesquisa transversal, descritiva e exploratória de caráter quantitativo, a ser realizado com as fichas de notificação compulsória dos óbitos por coronavírus, da vigilância epidemiológica do município de Foz do Iguaçu-PR. Os dados foram agrupamos em diversas categorias: sexo, idade, cor, nacionalidade, escolaridade, necessidade de internação, tipo de transmissão e morbidades. Desta forma, através deste estudo, identificou-se maior mortalidade no grupo masculino, de idade entre 70 a 79 anos, brancos, nacionalidade brasileira, baixa escolaridade, necessidade de internação, tipo de transmissão comunitária e entre as comorbidades foram mais presentes: hipertensão, IMC acima do adequado, diabetes, doenças cardiovasculares e pulmonares. Alguns indivíduos apresentaram uma ou mais comorbidade, a taxa de pessoas sem comorbidades ou em investigação foram baixas. Concluindo assim que esses fatores associados ao coronavírus mostraram influência na mortalidade desses indivíduos.
\end{abstract}

Palavras-chave: Coronavírus; Doenças crônica; Mortalidade.

\begin{abstract}
This study aims to analyze the factors associated with mortality by COVID-19 in a city of the triple border. This is a cross-sectional, descriptive and exploratory research of quantitative nature, to be carried out with the compulsory notification forms of deaths by coronavirus, the epidemiological surveillance of the municipality of Foz do Iguaçu-PR. The data were grouped into several categories: sex, age, color, nationality, education, need for hospitalization, type of transmission and morbidities. Thus, through this study, we identified higher mortality in the male group, aged between 70 and 79 years, white, Brazilian nationality, low education, need for hospitalization, type of community transmission and among the comorbidities were more present: hypertension, BMI above the appropriate, diabetes, cardiovascular and pulmonary diseases. Some individuals presented one or more comorbidity, the rate of people without comorbidities or under investigation were low. Thus concluding that these factors associated with the coronavirus showed influence on the mortality of these individuals.
\end{abstract}

Keywords: Coronavirus; Chronic diseases; Mortality.

\section{Resumen}

Este estudio pretende analizar los factores asociados a la mortalidad por COVID-19 en una ciudad de la triple frontera. Se trata de una investigación transversal, descriptiva y exploratoria de carácter cuantitativo, realizada con las fichas de notificación obligatoria de los óbitos por coronavírus, de la vigilancia epidemiológica del municipio de Foz do IguaçuPR. Los datos se agruparon en varias categorías: sexo, edad, color, nacionalidad, educación, necesidad de hospitalización, tipo de transmisión y morbilidad. Así, a través de este estudio, se identificó una mayor mortalidad en el grupo de varones, con edad entre 70 y 79 años, de raza blanca, nacionalidad brasileña, baja educación, necesidad de hospitalización, tipo de transmisión comunitaria y entre las comorbilidades estaban más presentes: hipertensión, IMC por encima del adecuado, diabetes, enfermedades cardiovasculares y pulmonares. Algunos individuos presentaban una o más comorbilidades, la tasa de personas sin comorbilidades o en investigación era baja. Por lo tanto, se concluye que estos factores asociados al coronavirus mostraron influencia en la mortalidad de estos individuos.

Palabras clave: Coronavirus; Enfermedades crónicas; Mortalidad. 


\section{Introdução}

O coronavírus é classificado como uma doença viral, denominado "Coronavírus da síndrome respiratória aguda grave 2" ou SARS-CoV-2, com origem na cidade de Wuhan, província de Hubei na China central, que se espalhou de forma rápida para mais de 180 países. Este vírus ocasionou um grande número de mortes, com milhares de casos confirmados em todo o mundo, representando uma série de ameaça à saúde pública (OMS, 2020).

Dados recentes mostram que cerca de $80 \%$ dos pacientes com COVID-19 podem ser assintomáticos ou oligossintomáticos (poucos sintomas), e aproximadamente $20 \%$ dos casos detectados requer atendimento hospitalar por apresentarem dificuldade respiratória, dos quais aproximadamente 5\% podem necessitar de suporte ventilatório (Brasil, 2020).

Os sintomas do COVID-19 podem variar de um simples resfriado até uma síndrome gripal (SG), ocasionando sintomas como: tosse, febre, coriza, dor de garganta, dificuldade para respirar, perda de olfato, alteração do paladar, distúrbios gastrintestinais (náuseas e vômitos), cansaço, diminuição de apetite e dispneia (falta de ar) (Brasil, 2020).

Ainda de acordo com Brasil (2020), por se tratar de um vírus de contaminação rápida e facilidade na sua transmissão, é importante se atentar às formas de contágio, que podem ocorrer por meio do toque ou aperto de mãos contaminadas, gotículas de saliva, espirro, tosse, catarro, objetos ou superfícies contaminadas, como celulares, mesas, talheres, maçanetas, brinquedos, teclados de computador, etc.

Algumas doenças podem ser classificadas como fatores de risco para a mortalidade ocasionada por esse vírus, como hipertensão, diabetes, doenças do sistema respiratório, doenças cardiovasculares e suas condições de suscetibilidade podem estar relacionadas à patogênese da COVID-19 devido aos mecanismos fisiológicos que desencadeiam diversas alterações no organismo, mantendo o indivíduo em vulnerabilidade (Yang et al., 2020).

O período de incubação da infecção por corona vírus é de cinco dias, com intervalo que pode chegar a 16 dias, o período de transmissão dos pacientes infectados é em sete dias após o início dos sintomas, os primeiros casos de transmissão foram notificados pela China, incluindo as regiões administrativas especiais de Hong Kong, Macau e Tapei, alguns desses casos confirmados apresentaram histórico de viagem, sendo assim, a transmissão do vírus alastrou-se de forma rápida, alcançando vários países pelo mundo, tornando-se uma pandemia (Brasil, 2020).

Observando essa característica de transmissão, conclui-se que as fronteiras e grandes metrópoles sofrem um impacto maior da doença, com isso levou ao fechamento de diversas fronteiras pelo mundo tornando-se importante novos estudos, que visam elucidar os mecanismos patogênicos e características epidemiológicas para identificar quais fatores contribuem para a mortalidade destas populações e que contribua para a informação e conscientização (Neto et al., 2020).

Diante do exposto, esse estudo objetiva analisar a correlação entre as comorbidades e fatores de risco que influenciam na mortalidade por coronavírus na cidade de Foz do Iguaçu-PR.

\section{Metodologia}

Estudo transversal retrospectivo em que foram analisados os dados referentes aos casos de óbitos decorrentes da COVID-19 atendidos e notificados em serviços de saúde de Foz do Iguaçu, Paraná, abrangendo o primeiro trimestre de 2021. Tal município faz parte de uma região de tríplice fronteira com Argentina e Paraguai.

A pesquisa foi de caráter censitário, utilizando dados contidos em fichas de notificação do Sistema Nacional de Agravos de Notificação (SINAN).

Nos casos de óbitos pela COVID-19, há uma ficha individualizada para tal doença, composta por um conjunto de variáveis específicas para caracterizar os óbitos em decorrência dessa enfermidade. A amostra foi composta por 381 fichas de notificação referentes ao primeiro trimestre de 2021. 
Foram analisadas as seguintes variáveis: sexo, idade, cor, nacionalidade, escolaridade, ocupação, tipo de transmissão, necessidade de internação e morbidades.

Este estudo se iniciou após a aprovação do Comitê de Ética em Pesquisa Envolvendo Seres Humanos (CEP), vinculado ao Conselho Nacional de Ética em Pesquisa (CONEP), respeitando todas as questões éticas e legais regidos nas resoluções CNS 466/2012 e CNS 510/2015, mantendo a integridade física e emocional, a dignidade e os interesses de todos os envolvidos na pesquisa. O projeto foi submetido ao CEP e aprovado sob Parecer n ${ }^{\circ} 5.053 .958$.

\section{Resultados e Discussão}

A amostra deste estudo foi composta por 381 notificações, de ambos os sexos, sendo $43 \%$ do sexo feminino (n=164) e $57 \%$ do sexo masculino $(\mathrm{n}=217)$ com idade a partir de 20 anos, que foram a óbitos na cidade de Foz do Iguaçu-PR no primeiro trimestre de 2021. Além de sexo e idade, foram obtidos dados sobre cor, nacionalidade, escolaridade, necessidade de internação, tipo de transmissão e morbidades.

A Tabela 1 representa a caracterização dos casos de óbito por COVID-19 quanto ao sexo, idade, cor, nacionalidade e escolaridade na cidade de Foz do Iguaçu, Paraná, Brasil.

Tabela 1. Caracterização dos casos de óbito por COVID-19 quanto ao sexo, idade, cor, nacionalidade e escolaridade. Foz do Iguaçu, Paraná, Brasil

\begin{tabular}{|c|c|c|}
\hline VARIÁVEIS & $\mathbf{N}$ & $\%$ \\
\hline \multicolumn{3}{|l|}{ SEXO } \\
\hline Feminino & 164 & 43 \\
\hline Masculino & 217 & 57 \\
\hline \multicolumn{3}{|l|}{ IDADE } \\
\hline 20 a 29 & 4 & 1 \\
\hline 30 a 39 & 13 & 3 \\
\hline 40 a 49 & 32 & 8 \\
\hline 50 a 59 & 51 & 13 \\
\hline 60 a 69 & 90 & 24 \\
\hline 70 a 79 & 124 & 33 \\
\hline Maior de 80 anos & 67 & 18 \\
\hline \multicolumn{3}{|l|}{ COR } \\
\hline Branca & 183 & 48 \\
\hline Preta & 9 & 2 \\
\hline Parda & 170 & 45 \\
\hline Não informada & 19 & 5 \\
\hline \multicolumn{3}{|l|}{ NACIONALIDADE } \\
\hline Brasil & 333 & 87 \\
\hline Paraguay & 11 & 3 \\
\hline Líbano & 4 & 1 \\
\hline Argentina & 3 & 1 \\
\hline Uruguai & 1 & 0,2 \\
\hline Bolívia & 1 & 0,2 \\
\hline Bangladesh & 1 & 0,2 \\
\hline Coréia do Sul & 1 & 0,2 \\
\hline Não informado & 26 & 7 \\
\hline \multicolumn{3}{|l|}{ ESCOLARIDADE } \\
\hline 1 a 2 anos & 102 & 27 \\
\hline 3 a 7 anos & 70 & 18 \\
\hline 8 a 11 anos & 73 & 18 \\
\hline 12 anos ou mais & 49 & 13 \\
\hline Não informada & 87 & 23 \\
\hline
\end{tabular}

Fonte: SINAN (2021). 
Analisando os dados das duas primeiras variáveis, que apresenta os índices de mortalidade quanto ao sexo e idade, observou-se no estudo conduzido por Fredrich, et al. (2021) que os indivíduos que evoluíram a óbito em sua maioridade eram do sexo masculino (68\%), nesse estudo foi encontrado dados similares, já que a incidência de óbitos neste grupo foi de $57 \%$. Isso acontece devido aos fatores sociais e culturais, como por exemplo falta de procura aos serviços primários de atenção à saúde, além da resistência na procura por tratamento, ou até mesmo as informações errôneas divulgadas por grupos isolados que não esse fator interfere na saúde desta população, justificando a maior mortalidade deste grupo.

Dados da Organização Mundial da Saúde (Who, 2020) sugere que o SARS-CoV-2 infecta pessoas de todas as faixas etárias, mas indivíduos com idade acima de 60 anos, juntamente com comorbidades como diabetes, doenças respiratórias crônicas e doenças cardiovasculares, têm maior risco de desenvolver infecção.

A taxa de mortalidade em pessoas com idade $\geq$ a 60 anos foi de $67 \%$, quando comparada a esse estudo se equivalem, já que $24 \%$ tem entre 60 a 69 anos, 33\% têm entre 70 a 79 anos e 18\% acima de 80 anos (Frederich, et al., 2021). Esses dados podem ser retratados pelo índice de maior comorbidades e fragilidade associado a imunosenescência aumentando o risco de óbitos nesse público. Outro fator que desse ver levado em consideração é a dificuldade cognitiva, de realizar as atividades diárias básicas o que prejudica no autocuidado, elevando o agravamento da doença podendo levar ao óbito (Wachholz, et al. 2020).

Referente a cor, percebeu-se que grande parte dos óbitos eram de indivíduos brancos (48\%), seguido de pardas (45\%). Frederich, et al. (2021) constataram que a taxa de mortalidade também foi maior em brancos, chegando a um percentual de $80 \%$. porcentagem entre pessoas brancas foi de $80 \%$ no estudo apresentado por em nosso estudo também teve em sua maioridade óbitos entre indivíduos de cor branca sendo eles $48 \%$. Fatores que podem ser observados nesta diferença vão desde maior prevalência de comorbidades, até um maior índice de pessoas negras trabalhando em serviços essenciais.

Um dado que chama a atenção é a nacionalidade dos indivíduos que evoluíram a óbito, pois percebe-se que a grande maioria (87\%) era brasileira, todavia, também foram constatadas nacionalidades paraguaias $(n=11)$, libaneses $(n=4)$ e argentinos $(n=3)$, dentre outras. Tais dados se justificam por Foz do Iguaçu fazer parte da maior região de tríplice fronteira do Brasil, assim como pelo alto número de turistas, o que aumenta a incidência de novos casos, sobretudo os importados. Durante a pandemia foram adotadas diversas medidas sanitárias para o controle da circulação de pessoas, tanto habitantes da cidade como estrangeiros. Foram criadas barreiras sanitárias em pontos estratégicos com verificação de sinais e sintomas respiratórios, fechamento de espaços e serviços que pudessem aglomerar alto número de pessoas, além do controle de circulação de pessoas em vias e/ou estabelecimentos.

Outra medida importante para a contenção do número de casos foi o fechamento das fronteiras terrestre com o Paraguai em março de 2020 e que deve duração de cerca de seis meses, com posterior abertura de forma parcial, com o controle sanitário necessário para a entrada no país. Já a Argentina manteve suas fronteiras fechadas por mais de 1 ano e seis meses, também com abertura de forma parcial e com um controle rigoroso de entrada de estrangeiros.

Nesta pesquisa nota-se uma maior prevalência de óbitos no público com escolaridade de 1 a 2 anos, chegando a 102 óbitos, já os indivíduos com escolaridade de 12 anos ou mais apresentou 49 óbitos, esse mesmo resultado foi encontrado no estudo realizado por Batista et al. (2020), as pessoas com nível de escolaridade superior apresentaram menor proporção de óbitos $(22,5 \%)$ que aquelas sem escolaridades $(71,3 \%)$.

A Tabela 2 apresenta a caracterização dos casos de óbito por COVID-19quanto a necessidade de internação, tipo de transmissão e morbidades. Percebeu-se que 362 indivíduos (95\%) necessitaram de cuidados hospitalares, enquanto 19 (5\%) não precisou. Vale ressaltar que a maioria dos casos graves necessitaram de internação e, portanto, era de se esperar que o número de óbitos dentre esse grupo seria maior. 
Tabela 2. Caracterização dos casos de óbito por COVID-19 quanto a necessidade de internação, tipo de transmissão e morbidades. Foz do Iguaçu, Paraná, Brasil

\begin{tabular}{|c|c|c|}
\hline VARIÁVEIS & $\mathbf{N}$ & $\%$ \\
\hline \multicolumn{3}{|c|}{ NECESSITOU DE INTERNAÇÃO } \\
\hline Sim & 362 & 95 \\
\hline Não & 19 & 5 \\
\hline \multicolumn{3}{|l|}{ TIPO DE TRANSMISSÃO } \\
\hline Comunitário & 365 & 96 \\
\hline Importado & 5 & 1 \\
\hline Local & 17 & 4 \\
\hline \multicolumn{3}{|l|}{ MORBIDADES } \\
\hline Sim & 359 & 94 \\
\hline Não & 21 & 6 \\
\hline Em investigação & 1 & 0,2 \\
\hline
\end{tabular}

Fonte: SINAN (2021).

Sobre o tipo de transmissão, os resultados indicaram que $96 \%(n=365)$ foram transmitidos através de transmissão comunitária, $1 \%$ importado $(n=5)$ e $4 \%(n=17)$ foi transmissão local.

Em relação as morbidades $94 \%(n=359)$ apresentaram comorbidade, $6 \%(n=21)$ não tinham comorbidade e 0,2\% (n=1) estavam em investigação.

A extração dos dados conferiu 157.268, resultando na amostra final de 29.933 casos que foram a óbito por covid 19. Considerando o tipo de internação, têm-se que 30,4\% dos pacientes em enfermaria vieram a óbito, enquanto que nos casos em UTIs esta proporção foi de 65,3\% (Batista, et al., 2020), dados similares foram analisados em nosso estudo já que o número de indivíduos que precisaram de internamento foram 362, representando $95 \%$.

O Ministério da Saúde declarou através da Portaria n454, de 20 de março de 2020, o reconhecimento da transmissão comunitária do coronavírus Covid-19 em todo o território nacional (Brasil, 2020). Mesmo com as medidas sanitárias implementadas, afim de controlar o índice de transmissão, nesse presente estudo notou se que o percentual de transmissão comunitária foi de $96 \%$, confirmando o reconhecimento do ministério da saúde sobre o avanço da contaminação por covid 19.

Embora tenha se passado dois anos após o primeiro caso de COVID-19, o conhecimento das características básicas do paciente e dos fatores de risco associados à UTI e à mortalidade hospitalar ainda não é totalmente ilucidado. Sexo masculino, hipertensão, doenças cardiovasculares e diabetes tipo 2 são as comorbidades mais prevalentes, e estão associadas a uma alta taxa de letalidade por casos (Grasselli, et al., 2020).

Um estudo conduzido por Ramos (2020), mostrou que os óbitos com comorbidades associadas somaram 1.452 casos $(82,17 \%)$ e aqueles sem comorbidades associadas totalizaram 315 casos (17,83\%). Em nosso estudo encontramos dados similares, já que 359 (94\%) foram a óbito com uma ou mais comorbidades, e 21 (6\%) foram a óbito sem apresentar nenhuma comorbidade, ainda $1(0,2 \%)$ estava em investigação.

Este presente estudo identificou uma maior mortalidade no público que apresenta comorbidades, entre elas as mais presentes são: hipertensão $(n=279)$, diabetes $(n=125)$, IMC acima do saudável $(n=117)$, doenças cardiovasculares $(n=92)$ e doenças pulmonares $(n=52)$. Quando analisados diversos outros estudos, observamos um pior prognósticos, maior mortalidade e severidade dessa doença em pacientes que apresentam comorbidades.

Estudos que investigam os prováveis mecanismos moleculares, fisiológicos e vias metabólicas que os relacionam com o coronavírus e as comorbidades ainda são escassos, porém alguns achados podem fornecer possíveis justificativas dos elementos 
centrais dessa relação, três elementos têm ganhado destaque: 1: inflamatório; 2: enzimas conversoras de angiotensina 2 (ECA2); e 3: hiperglicemia. Isso ocorre porque no organismo desses pacientes podem haver alterações nas células, prejudicando o metabolismo, como a hiperglicemia, que também está diretamente relacionada a uma menor resposta imune e maior cetoacidose diabética durante as infecções, o que prejudica a resposta do organismo (Souza, et al. 2020).

Pessoas com diabetes estão inclinadas a contraí-los devido a capacidades celulares fagocíticas prejudicadas. Além disso, vários outros fatores aumentam o risco de COVID-19 em pacientes diabéticos (Rao, et al., 2020). Um nível elevado de receptores ACE-2 encontrados causalmente relacionados ao diabetes pela análise de randomização mendeliana; isso pode prejudicar as pessoas com diabetes à infecção pelo SARS-CoV-2 (Wang, et al., 2020).

Dados emergentes sobre o COVID-19 sugerem que 11-58\% de todos os pacientes COVID-19 têm diabetes, e uma taxa de fatalidade de $8 \%$ COVID-19 foi relatada em pacientes diabéticos (Yang, et al., 2020). O risco de internações em UTI em indivíduos COVID-19 com comorbidade diabética é 14,2\% maior do que indivíduos sem diabetes.

Outro elemento importante na resposta do organismo durante a infecção por corona vírus é a enzima conversora de angiotensina 2 é considerado um eixo central na regulação da pressão arterial, os estudos sugerem que o ECA2 são vias contra regulatórias e importantes protetoras vasculares e pulmonares, demostrando ser um elemento importante na resposta do organismo contra o coronavírus (Souza, et al. 2020).

Além da hiperglicemia e a resposta do ECA2, o sobrepeso e obesidade também são elementos importantes na relação das comorbidades e o coronavírus, isso porque em pacientes obesos, além da inflamação do organismo. esses indivíduos demonstram necessidade aumentada ao suporte de oxigênio, além disso, esses indivíduos apresentam aumento de adipócitos, podendo servir como depósito viral, onde alguns vírus tem a habilidade de invadir adipócitos, podendo ser a porta de entrada para o coronavírus (Souza, et al. 2020). A obesidade não foi um fator de risco para o COVID-19 nos primeiros relatórios da China, Itália e Estados Unidos (Grasselli, et al., 2020). No entanto, o alto número de casos DE COVID-19 observados nas regiões com mais obesos da Europa e América do Norte (Ryan, et al., 2020). Assim, é necessário explorar a relação da obesidade com a frequência do COVID-19. A obesidade é uma das comorbidades menos destacadas nas infecções pelo o COVID-19. Esses estudos comprovam o prejuízo da resposta imunológica em paciente que apresentam comorbidades, contribuindo para o pior prognóstico de mortalidade pela COVID-19.

Doenças subjacentes, como hipertensão arterial, DCV, diabetes, malignidade, DPOC e asma, têm sido relatadas como fatores de risco para doenças graves e também aumentaram a taxa de mortalidade, portanto, uma melhor gestão com especial consideração deve ser dada a esses pacientes. A maioria dos pacientes do COVID-19 morre devido à comorbidade pré-existente; portanto, é necessária uma avaliação precisa no momento da internação (Ejaz, et al., 2020).

\section{Considerações Finais}

O município de foz do Iguaçu apresentou um número significativo de óbitos por COVID-19 no primeiro trimestre de 2021, levando em consideração que pode existir ainda subnotificações, o que dificulta na análise dos dados obtidos.

A infecção pelo COVID-19 torna-se prejudicial quando confronta uma pessoa idosa e com comorbidade, a necessidade do manejo desses pacientes com cuidados hospitalares adequados é um passo imprescindível para sua sobrevivência. Prestar esse atendimento em um nível de alta qualidade para o alto volume de pacientes para tratar é um desafio para todos os sistemas de saúde.

\section{Referências}

Batista, A., Antunes, B., Faveret, G., Peres, I., Marchesi, J., Cunha, P. J., Dantas, L., Bastos, L., Carrilho, L., Aguilar, S., Baião, F., Maçaira, P., Hamacher, S., \& Bozza, F. (2020). Análise socioeconômica da taxa de letalidade da COVID-19 no Brasil https://www.sites.google.com/view/noispucrio/publica\%C3\%A7\%C3\%B5es?authuser=0 
Carneiro, J; \& Allis, T. (2021). Como se move o turismo durante a pandemia da COVID-19? Revista Brasileira de Pesquisa em Turismo, 15 (1), 1-23.

Ejaz, H., Alsrhani, A., Zafar, A., Javed, H., Junaid, K., Abdalla, A. E., Abosalif, K. O. A., Ahmed, Z., \& Younas, S. (2020). COVID-19 and comorbidities: Deleterious impact on infected patients. J Infect Public Health. 13 (12): 1833-1839.

Fredrich, V. C., Asr, A. M., Champion, L., Mello, T., Silva, J. V., Ziak, M., Bellizzi, A., Lopes, M., Junior, N., \& Preto, C. (2020). Perfil de óbitos por covid-19 no estado do paraná no início da pandemia: estudo transversal. Revista de saúde pública do paraná, 3(1), 62-74.

Grasselli, G., Greco, M., Zanella, A., Albano, G., Antonelli, M., Ballani, G., et al. (2020). Risk Factors Associated With Mortality Among Patients With COVID19 in Intensive Care Units in Lombardy, Italy. JAMA Intern Med. 180(10): 1-11.

Grasselli, G., Zangrillo, A., Zanella, A., Antonelli, M., Cabrini, L., \& Castelli A. (2020). Baseline characteristics and outcomes of 1591 patients infected with SARS-CoV-2 admitted to ICUs of the Lombardy region, Italy. JAMA. 323(16):1574-1581.

Melo, R. R. (2020). Análise do perfil epidemiológico dos óbitos por COVID-19 em Santa Catarina durante a pandemia de coronavírus até a 33a semana epidemiológica do ano de 2020 https://repositorio.ufsc.br/handle/123456789/218111.

Mizumoto, K., Kagaya, K., Zarebski, A., \& Chowell, G (2020). Estimating the asymptomatic proportion of coronavirus disease 2019 (COVID-19) cases on board the Diamond Princess cruise ship, Yokohama, Japan, 2020. Euro Surveill, 25(10), 1-5.

Neto, O. T., Garcia, L. S. T., \& Spinussi, E., (2020). Pandemia de COVID-19, as fronteiras pelo mundo e o transporte aéreo na Itália https://journals.openedition.org/confins/27577

Opas. Organização pan-americana de saúde (2021). Histórico da pandemia de covid 19. S.D. https://www.paho.org/pt/covid19

Rao, S., Lau, A., \& So H. C. (2020). Exploring diseases/traits and blood proteins causally related to expression of ACE2, the putative receptor of SARS-CoV2: a Mendelian randomization analysis highlights tentative relevance of diabetes-related traits. Diabetes Care.

Ryan, D.h., Ravussin, E., \& Heymsfield, S. (2020). COVID 19 and the patient with obesity-the editors speak out. Obesity. 28(5):847.

Sahebnasagh, A., Avan, R., Saghafi, F., Mojtahedzadeh, M., Sadremomtaz, A., Arasteh, O., Tanzifi, A., Faramarzi, F., Negarandeh, R., Safdari, M., Khataminia, M., Ghaleno, R. H., Habtemariam, S., \& Khoshi, A. (2020). Pharmacological treatments of COVID-19. O Pharmacol, 72, 1446-1478.

Singh, K. A., Gupta, R., \& Misra, A. (2020). Comorbidities in COVID-19: Outcomes in hypertensive cohort and controversies with renin angiotensin system blockers. Sciencedirect, 14(4), 283-287.

Souza, A. T., Siqueira, S. B., \& Grassiolli, S. (2020). Obesidade, Comorbidades e Covid19: Uma breve revisão de literatura. Ciências Da Saúde, 6(1), 72-82.

Streeck, H. et al. Infection fatality rare of SARS-CoV2 in a super-spreading event in Germany. Rev. Nature Communications. Nov, 2020.

Wachholz, A. P., Moreira, G. V., Oliveira, D., Watanabe, H., \& Boas, V. F. J. P (2016). Ocorrência de infecção e mortalidade por covid-19 em casas de idosos no brasil https://preprints.scielo.org/index.php/scielo/preprint/view/1032/version/1096

Wang, D., Hu, B., Hu, C., Zhu, F., Liu, X., \& Zhang J. (2020). Clinical characteristics of 138 hospitalized patients with 2019 novel coronavirus-infected pneumonia in Wuhan, China. JAMA. 323(11):1061-1069.

Who. World Health Organization (2020). Coronavirus disease 2019 (COVID-19) Situation report - 141. Geneva, Switzerland: https://www.who.int/docs/default-source/coronaviruse/situation-reports/20200609-covid-19-sitrep-141.pdf?sfvrsn=72fa1b16_2.

Who. World Health Organization (2021). Infection prevention and control during health care when COVID-19 is suspected https://www.who.int/publications/i/item/WHO-2019-nCoV-IPC-2021.1

Yang, J., Zheng, Y., Gou, X., Pu, K., Chen, Z., \& Guo Q. (2020). Prevalence of comorbidities in the novel Wuhan coronavirus (COVID-19) infection: a systematic review and meta-analysis. Int J Infect Dis. 94:91-95.

Yang, J., Zheng, Y., Gou, X., Pu, K., Chen, Z., Guo, Q., Ji, R., Wang, H., Wang, Y., \& Zhou, Y. (2020). Prevalence of comorbidities and its effects in patients infected with SARS-CoV-2: a systematic review and meta-analysis. International journal of infectious diseases: IJID: official publication of the International Society for Infectious Diseases, 94, 91-95. 